\title{
Significado de la calidad de vida del adulto mayor institucionalizado en un albergue, Lima 2011
}

\author{
Martha Vera, Rocío Cornejo, Fabiola Quiroz, Jessica Calizaya, Gloria Torrelli, \\ Geraldine Tasayco \\ Instituto de Ética en Salud, Facultad de Medicina, UNMSM.
}

Objetivos: Interpretar el significado de la calidad de vida del adulto mayor institucionalizado.

Diseño: Estudio cualitativo, descriptivo - interpretativo, fundamentado en la Teoría de las Representaciones Sociales.

Institución: Instituto de Ética en Salud, Facultad de Medicina, UNMSM.

Participantes: Adultos mayores.

Intervenciones: A 30 adultos mayores, 18 mujeres y 12 hombres, del Albergue Canevaro, Lima, grupo etario predominante de 66 a 80 años, durante tres meses se aplicó entrevista a profundidad y guía estructurada con seis preguntas norteadoras; se preguntó el significado de la calidad de vida del adulto mayor.

Principales medidas de resultados: Descontextualización de los discursos, codificación y emergencia de categorias.

Resultados: Después del análisis ideográfico - axial, la objetivación y el anclaje emergieron como representación social, con dos unidades de significado (categorías): conocimiento acerca de la vejez y ser un adulto mayor.

Conclusiones: La calidad de vida del adulto mayor significaba estar y tener a Dios como su protección, pues lo hacía sentir bien, satisfecho, tendiendo a la tranquilidad y paz. Era tener satisfechas sus necesidades físicas, emocionales y sociales, aunque con las limitaciones propias de la edad, teniendo beneficios que nunca tuvieron en su vida fuera de la institución. Tener el tiempo suficiente para seguir siendo útil, seguir trabajando.

Palabras clave: Calidad de vida, adulto mayor, representación social, institucionalizado.

\section{Predictores del síndrome de burnout en estudiantes de segundo año de las Escuelas de Medicina y Nutrición de la Facultad de Medicina de la UNMSM 2011}

\author{
Ana Delgado \\ Facultad de Medicina, UNMSM
}

Objetivos: Determinar los predictores del síndrome de burnout (SB) en estudiantes de segundo año de las Escuelas de Medicina y Nutrición. Identificar su presencia en dichos estudiantes, las tres dimensiones que lo conforman como sus predictores y sus características socio demográficas.

Diseño: Estudio observacional, analítico, transversal.

Institución: Facultad de Medicina, UNMSM.

Participantes: Estudiantes de segundo año de las Escuelas de Medicina y Nutrición.

Intervenciones: Se aplicó un cuestionario cerrado con gradación, que incluyó características sociodemográficas y laborales, así como una adaptación del MBI-SS (Maslach Burnout Inventory-Student Survey) de Schaufeli y colaboradores (2002), para determinar la presencia o no del SB y sus predictores.

Principales medidas de resultados: 30 ítems distribuidos en 3 escalas: agotamiento emocional, despersonalización y baja realización personal.

Resultados: El mayor riesgo de SB estuvo en los estudiantes de nutrición (6,7\%). Las dos primeras escalas tuvieron puntuaciones elevadas en $26,3 \%$ de casos: $16,2 \%$ a medicina en la primera y $2,7 \%$ a nutrición en la segunda. Ninguno obtuvo puntuación alta en las 2 escalas a la vez.

Conclusiones: Si bien las tres escalas no presentaron valores indicativos de la presencia del síndrome en los estudiantes, se encontró niveles bajos en realización personal en $51,7 \%$ de estudiantes (55,5\% medicina). El comportamiento de los predictores del SB se asoció con la edad (22 a 26 años), género (masculino), estado civil (casados y convivientes), presencia de hijos y en los que trabajan.

Palabras clave: Predictores, síndrome burnout, estudiantes de segundo año, Medicina, Nutrición. 